\title{
Modeling on the Interaction of the Turbulent Diffusion Flames between a Vertical Burning Wall and a Pool Fire
}

\author{
H.Y. WANG and P. JOULAIN \\ Laboratoire de Combustion et de Détonique \\ C.N.R.S. UPR 9028 -E.N.S.M.A., Université de Poitiers \\ BP 109-Site du Futuroscope, 86960, Futuroscope Cedex, France
}

\begin{abstract}
Until now, very few works have been devoted to the predictions of the flame structure resulting from the combustion of a vertical burning wall adjacent to a rectangular pool fire at the floor level. This paper describes the application of a three-dimensional model to the prediction of the aerodynamic field and the thermal structure using a finite volume method for solving the fluid dynamic equations. The model employs a two-equations, $\mathrm{k}-\varepsilon$ turbulence model. The gas-phase, non-premixed combustion process is modeled via the conservated scalar/prescribed density function approach. It can be indicated that the merging of the diffusion flames between a vertical burning wall and a pool fire at the floor level induces a rapid broadening of the buoyant gas column. As a consequence, the flame thickness remains almost constant all along the vertical burning wall, and the buoyantly induced plume closer to the horizontal combustible surface is strongly narrowed. Predictions are shown to be in reasonable agreement with measurements and areas requiring further research are selected.
\end{abstract}

KEYWORDS: modeling, vertical burning wall, pool fire, turbulence, diffusion flame

\section{NOMENCLATURE}

f mixture fraction

$\mathrm{g}$ the acceleration of gravity parallel to the co-ordinate direction $\mathrm{x}$

$\mathrm{H}$ height of the vertical burning wall
W distance between the walls in the co-ordinate direction $\mathrm{z}$ 
$\mathrm{H}_{\mathrm{c}} \quad$ heat-released by combustion of unit mass of fuel

k turbulent kinetic energy

L length of the horizontal burning surface

$\dot{\mathrm{m}}_{\mathrm{s}} \quad$ surface mass rate of injection gas from the wall

s stoichiometric ratio

S source term

$\mathrm{T}$ mean temperature

$\mathrm{u}, \mathrm{v}, \mathrm{w}$ velocity components in the co-ordinate directions $\mathrm{x}, \mathrm{y}$ and $\mathrm{z}$ respectively

\section{Greek symbol}

$\varepsilon \quad$ dissipation of the turbulent kinetic energy

$\rho$ density

$\phi \quad$ general variable

$\Gamma \quad$ diffusion coefficient

\section{Subscripts}

eff effective value

$\phi \quad$ relative to the variable $\phi$

\section{INTRODUCTION}

This work presents a computational fluid dynamics simulation of an experimental fire developing along a vertical burning wall adjacent to a rectangular pool fire at the floor level. Until now, most studies have been focused on either a vertical burning wall [1-5] or a pool fire [6-9]. The typical studies mentioned here show that the flame thickness along a vertical burning wall increases linearly with height, whilst the flame height of a pool fire is proportional to its size and heat release rate. Comparatively, less fundamental work has been conducted on the interaction of the turbulent diffusion flames between a vertical burning wall and a pool fire at the floor level. To the best knowledge of the authors, only the experimental work of Annarumma [8] has addressed such wall-pool fire, but which is not readily described by a mathematical analysis. Therefore, the objective of this work is to develop a predictive capacity for quantifying the influence of a pool fire on the flame shape and flow field developing along a vertical burning wall.

During such wall-pool fire, flame developing over combustible surfaces is characterized by a very low initial momentum. The gas flow is usually buoyantly induced by density gradients near the fire, and the turbulence is naturally generated. According to the experiment, the flame near the floor level appears to be laminar, and the flow field is displaced in a flapping manner due to a strong buoyant instability. The use of the k- $\varepsilon-\mathrm{g}$ model to calculate the distribution of Reynolds stresses for this flow situation, is not ideal, but is currently applicable to date everywhere as computational tools to solve numerically the fluid dynamic equations for a three-dimensional elliptic, reacting flow. Moreover, no turbulence model, up to now, is capable of yielding realistic predictions of all components of the Reynolds and turbulent scalar flux in the presence of a buoyant instability and a transition to turbulence. The turbulent nonpremixed combustion process is modeled using the fast chemistry assumption, together with a specified probability density function (pdf) for a single conserved scalar variable (mixture fraction) coupled to solutions of modeled transport equations for the mean and variance of that variable. Finally, validation has been possible by comparing the predictions with the experimental data [8] obtained from a laboratory scale release. The relatively good trend for predicting the general characteristic of the diffusion flame and flow field in an intermediate-scale vertical burning wall adjacent to a rectangular pool fire clearly proved the relevance for future development. 


\section{EXPERIMENT CONSIDERED}

A study of the influence of a turbulent diffusion flame on solid fuel burning rate is of particular importance. However, in gas/solid combustion, the experiment duration is always too short to characterize the fire behavior above the solid surface. Therefore, the burning of a combustible vertical surface, $1 \mathrm{~m}$ high $(\mathrm{H})$, adjacent to a rectangular pool fire with a crosssection ( $\mathrm{L} \times \mathrm{W}$ ) of $0.25 \times 0.4 \mathrm{~m}^{2}$, is simulated by injecting a gaseous fuel through a porous wall into the buoyancy-induced air stream. A schematic diagram of this wall-pool fire is shown in Figure 1, and a complete description of the experimental equipment and fire tests conducted is given in Ref. [8]. Turbulence and velocity were measured with a LDV system, and mean temperatures were obtained by means of fine wire analogue compensated thermocouples.

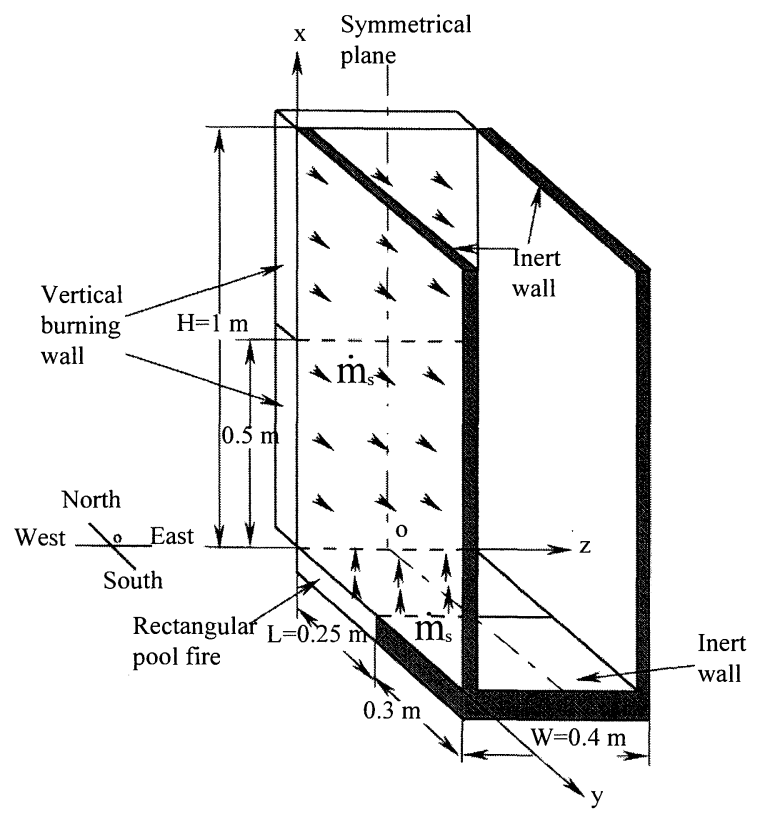

FIGURE 1. Schematic diagram of a vertical burning wall adjacent to a rectangular pool fire

The constants for heat of reaction, and oxygen/fuel stoichiometric ratio are respectively taken as $\mathrm{H}_{\mathrm{c}}=4.64 \times 10^{7} \mathrm{~J} / \mathrm{kg}$, and $\mathrm{s}=3.63$. The fuel (propane, $\mathrm{C}_{3} \mathrm{H}_{8}$ ) injection rate, $\dot{\mathrm{m}}_{\mathrm{s}}\left(\mathrm{kg} / \mathrm{m}^{2} \mathrm{~s}\right)$, is set at 0.0031 in the upper part of the wall $(0.5 \mathrm{~m})$ and at 0.0028 in the lower one (also $0.5 \mathrm{~m}$ ), corresponding to the range of the pyrolysis mass transfer rates of real combustible materials. The pool fire is stabilized over the horizontal porous plate, as shown in Figure 1, with an injection rate of 0.0053 . Of course, this mathematical model does not restrict its range of applicability to the case considered here, other fire configurations can also be considered. 


\section{THEORETICAL MODEL}

The basis of the mathematical model employed has been described in detail elsewhere [1-3]. In the following sections, a brief description of the turbulence and combustion models is presented. The perfect gas equation, the variation of viscosity with temperature and a number of other assumptions are also included in the model. Finally, a set of known boundary conditions is specified to complete the mathematical description.

\section{Governing Equations}

For steady flow, the mathematical model is therefore based on solutions of the threedimensional, partial differential conservation equations for mass, momentum and conserved scalar transport. The turbulent momentum and scalar fluxes that appear in the above equations are approximated by a standard two-equations, k- $\varepsilon$ turbulence model [10] that solves transport equations for turbulent kinetic energy and dissipation rate of turbulence energy. The governing differential equations may be written in a general cartesian formulation as :

$$
\frac{\partial}{\partial x}(\rho u \phi)+\frac{\partial}{\partial y}(\rho v \phi)+\frac{\partial}{\partial z}(\rho w \phi)=\frac{\partial}{\partial x}\left[\Gamma_{\phi, e f f} \frac{\partial \phi}{\partial x}\right]+\frac{\partial}{\partial y}\left[\Gamma_{\phi, \text { eff }} \frac{\partial \phi}{\partial y}\right]+\frac{\partial}{\partial z}\left[\Gamma_{\phi, \text { eff }} \frac{\partial \phi}{\partial z}\right]+S_{\phi}
$$

For the naturally generated flow, non-uniform buoyancy forces are allowed to affect both the mean and fluctuating flows. The effect of gravity on the mean flow is incorporated through the momentum equation in the x-direction. The influence of the buoyancy term, accommodated in the model of Ince [11] in which the normal diffusion gradient is taken into account, on the turbulence structure is also considered. To close the fluid dynamic equation, the local values of $\Gamma_{\phi, \text { eff }}$ is calculated from the $\mathrm{k}$ and $\varepsilon$. The empirical constants used in the model are assigned to the standard values.

The nonpremixed combustion process is modeled by assuming that the rate of reaction of fuel and air is controlled by the relatively slow turbulent mixing process and not by the fast reaction kinetics. So a simple one-step reaction is a fair one for this mixing-controlled or diffusion flame combustion process. All molecular species can be assumed to have the same diffusion coefficients, and the Lewis number is unity. Using the Shvab-Zeldovich formulation, the linear combination of the partial differential equations of the mean oxygen and fuel mass fraction is normalized for obtaining a conserved scalar, $f$, the mixture fraction which varies between 0 in the oxidizer flow to 1 in the fuel. The effect of turbulence on the combustion process is taken into account by weighting the mass fractions with a probability density function (pdf). A rectangular form of the pdf is chosen, which is specified in terms of the mean mixture fraction and its variance obtained from solution of modeled transport equations for these variables. Any scalar quantity that is uniquely related to mixture fraction can be evaluated by the Favre pdf for the mixture fraction.

In general, the predicted temperature is well above the measured peak one due to the lack of radiation and finite-rate chemistry in the model. To obtain more realistic temperatures, the heat-release parameter $\left(\mathrm{H}_{\mathrm{c}}\right)$ is reduced by 40 percent. A more accurate treatment for 
radiation is currently being developed which uses a three-dimensional adaptation of the discrete transfer method.

\section{Boundary Conditions}

With respect to Figure 1, the flow is symmetric about the $x-y$ plane located at $z=0.2 \mathrm{~m}$. The domain to be studied will be reduced to a half with zero gradients of all dependent variables normal to the plane being considered $(\mathrm{z}=0.2 \mathrm{~m})$, and with no flow across the plane.

At the inert solid surfaces, the no-slip condition is imposed by setting all velocities to zero. The gradient of the mixture fraction and of its fluctuation is set to zero at all boundaries due to the impermeability of the walls. However, the mixture fraction at the combustible solid surfaces is set according to the mass conservation [5]. Caution is required in applying the common $\mathrm{k}-\varepsilon$ turbulence model near all solid surfaces (inert or combustible) because of doubts over its reliability in highly non-isotropic flows. Therefore, the velocity component parallel to the wall concerned, the turbulent kinetic energy and the turbulence dissipation rate are estimated through a local equilibrium wall log-law. In the experiment, the temperature of the porous and inert walls is maintained respectively to about $800 \mathrm{~K}$ and $350 \mathrm{~K}$, using cooling water for the protection of the material. Correspondingly, the enthalpy is imposed through the given wall temperature when solving an energy conservation equation.

The south free boundary at $\mathrm{y}=0.55 \mathrm{~m}$ of the solution domain is treated as entraining surface on which ambient pressure is set to be constant. It is located sufficiently far away from the fire for the boundary conditions associated with this surface to have a negligible influence on the computed flow. Velocity components in this plane are set to zero and the normal velocity is determined from the continuity equation. While the high free boundary at $x=1.0 \mathrm{~m}$ is taken as outflow surface, normal gradients of all dependent variables are set to zero on the downstream plane. It is certain that this treatment at the high free boundary where the flow conditions are not well known, in the computational domain may influence the calculation of the entrainment, but this boundary can not be located infinitely far away from the physical domain due to the geometry effects on the flow structure and hardware limitations.

\section{Method of Resolution}

The finite volume method developed by Patankar [12] is used to discretize the partial differential equations. This procedure entails the subdividing of the calculation domain into a finite number of control volumes that employed a grid in cartesian coordinates, and a staggered arrangement in order to prevent uncoupling between pressure and velocity fields. The mean flow equations are numerically solved by using the SIMPLEC procedure [13] for handling the pressure- velocity coupling. A higher density of grid nodes is generated using a series distribution close to the wall. The grid is relatively fine (typically $57 \times 56 \times 20$ ), and grid dependence of the model solutions was examined. In terms of the mean parameters considered, further grid refinement would not have affected the flame structure and flow field. Convergence is achieved when the normalized residuals are less than $5 \times 10^{-3}$ for each equation with about 3000 iterations due to the strong coupling between the temperature and velocity 
fields. Global execution time on the DEC3100/alfa for obtaining a correct buoyancy-induced flow is about 8 hours.

\section{RESULTS AND DISCUSSION}

The general characteristic of the flame shape and flow field structure from the simulation is described, after which the influence of the pool fire on the vertical burning wall is discussed. The two features of the experiment [8] used to assess the results from the simulation are the velocity and temperature profiles and the turbulence intensity.

\section{Interaction between a Vertical Burning Wall and a Pool Fire}

The predicted temperature contours and velocity vectors on the symmetrical plane ( $\mathrm{x}$, $\mathrm{y}, \mathrm{z}=0.2 \mathrm{~m}$ ) are shown in Figures 2 and 3. The visible flame shape in Fig. 2 corresponds to the zone where the temperature is higher than $650 \mathrm{~K}$, and only the maximum velocity at each given elevation along the vertical burning wall is indicated in Fig. 3. It is seen that combustion is initially taking place near the edge of the horizontal burner (pool fire), where initial mixture between combustible gases and air is occuring. Under free convection conditions, ambient air flows strongly inward toward the reactive zone of the pool fire, and accelerates progressively upward along the vertical burning wall due to buoyancy forces produced by density stratification. Consequently, the flow is almost horizontally directed against the vertical wall due to a large horizontal air inflow and very low upward gas velocities. While fuel vapor flows away and up from the combustible surface due to the outwardly flowing fuel vapor. Finally, the fuel vapor and air move upward, converging toward each other following a converging streamline that moves progressively away from the vertical burning wall with the distance downstream.

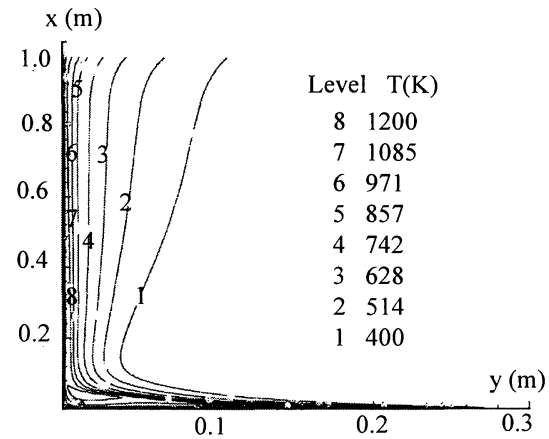

FIGURE 2. Temperature contours on the symmetrical plane $(\mathrm{x}, \mathrm{y}, \mathrm{z}=0.2 \mathrm{~m})$ in a perspective view from the west

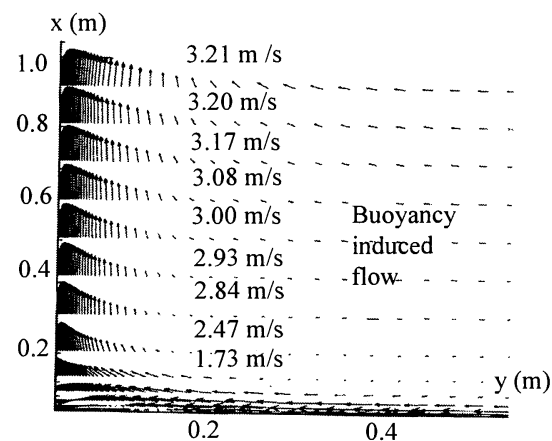

FIGURE 3. Velocity vectors on the symmetrical plane $(\mathrm{x}, \mathrm{y}, \mathrm{z}=0.2 \mathrm{~m})$ in a perspective view from the west

The flame shape and flow field structure developing along the vertical burning wall are extremely sensitive to the presence of the pool fire. The important features of these results in 
comparison with a similar single vertical burning wall [8] are as follows.

1. For a single vertical burning wall, a linear dependence of the flame thickness $\left(\mathrm{d}_{\mathrm{f}}=0.07 \mathrm{x} \mathrm{m}[8]\right)$, which is almost neglected at the leading edge, to the height is observed.

2. In the presence of a pool fire, the flame thickness at the base of the vertical burning wall, where the two diffusion flames developing along the vertical and horizontal combustible walls merge, is important. As a consequence, the flame maintains this advantage at distant downstream locations $(x>0.1 \mathrm{~m})$ with an almost constant thickness $\left(\mathrm{d}_{\mathrm{f}} \approx 0.07 \mathrm{~m}\right.$, experimental data). Note that the theoretical prediction, as shown in Fig.2, underestimates the flame thickness by about 40 percent $\left(\mathrm{d}_{\mathrm{f}} \approx 0.04 \mathrm{~m}\right)$, but with a similar flame shape to the experimental one.

3. The buoyancy-induced flow is rather weak at the base of a single vertical burning wall, and then accelarated more and more strongly, up to achieve a maximum velocity of $2.8 \mathrm{~m} / \mathrm{s}$ [8] at a height of $1 \mathrm{~m}$.

4. On the contrary, the buoyancy-induced flow along the vertical burning wall is significantly reinforced by the presence of a pool fire. At the base of the vertical wall, buoyancy effects are extremely important, and the flow is strongly upward accelerated up to $1.73 \mathrm{~m} / \mathrm{s}$ at a height of $0.1 \mathrm{~m}$. Then, at distant downstream locations $(x>0.1 \mathrm{~m})$, the velocity vectors which are very similar to that of a single vertical wall, become almost parallel to the vertical burning wall, and the acceleration of the flow increases progressively up to $3.21 \mathrm{~m} / \mathrm{s}$ at a height of $1 \mathrm{~m}$.

5. Finally, in the presence of the pool fire, the air entrainment at the base of the vertical wall is stronger than that of a single vertical wall. Then, far downstream away from the low base, the air entrainment decreases progressively with a linear dependence of the height first and later increases above a height of $0.4 \mathrm{~m}$ due to the increase of the hot gas boundary layer (see Fig. 2).

Also, the flame shape and flow field structure of the pool fire are significantly dependent on the vertical burning wall. For a clear view, the velocity vectors plot is presented in Figure 4 illustrating a largely stagnant area just above $(x=0.015 \mathrm{~m})$ the horizontal burner surface where the flow reversal is occurring with a large recirculation zone. As a consequence, a diffusion flame is stabilised over a larger zone $(0<\mathrm{y}<0.2 \mathrm{~m})$, as evidenced in Fig. 2 by the high temperature $(\mathrm{T}>650 \mathrm{~K})$ distribution near the low base, in comparison with that $(0<\mathrm{y}<0.1$ $\mathrm{m}$ ) for a similar pool fire in stagnant atmosphere [8]. Moreover, far downstream away from the pool fire $(x>0.1 \mathrm{~m})$, a rapid broadening of the buoyant gas column (see Fig. 3), which is almost horizontally directed against the vertical wall, results in a strong narrowing of the flame (see Fig. 2). This is due to the fact that the entraining ambient air at large mass flow rate tends to reduce the normal diffusion of heat at the floor level and moves the reaction zone closer to the horizontal combustible surface.

The three-dimensional nature of the temperature and flow fields is examined in Figures 5 and 6 on the plane $(\mathrm{x}, \mathrm{z})$ near the vertical burning wall $(\mathrm{y}=0.01 \mathrm{~m})$. The distribution of the temperature, as shown in Figure 5, is in a complex three-dimensional manner. The temperature profiles are stratified with the hottest gas located in the boundary layer near the walls, and almost uniform over most of the cross-section. Correspondingly, the threedimensional flow, as shown in Figure 6, is confined only to the corner region formed by the horizontal and vertical walls. The air flow deflects locally inward, toward the inert wall (west) 
due to a strong acceleration of the flow in the low corner region. Outside of this region, the flow is essentially two-dimensional, as evidenced by a uniform parallel flow between the vertical inert walls (west and east). An illustration of the temperature contours and velocity vectors field on the plane $(y, z)$ just above the horizontal burner surface $(x=0.015 \mathrm{~m})$ is also presented in Figures 7 and 8 . In the entrance region $(y>0.2 \mathrm{~m})$, the temperature stratification is seen to be almost negligible, and the flow is also practically two-dimensional in the sense that
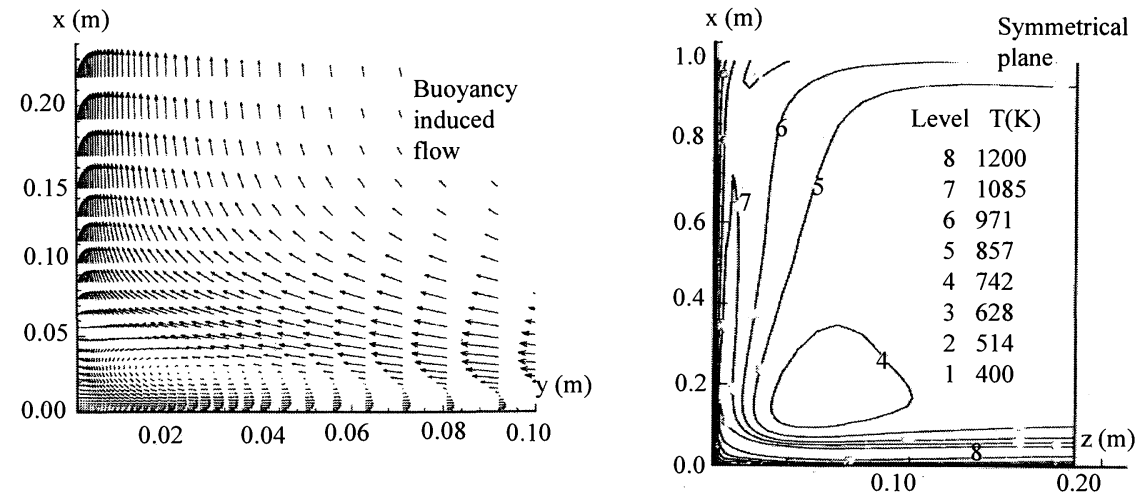

FIGURE 4. Velocity vectors near the corner fire formed by the vertical and horizontal burning walls on the symmetrical plane $(x, y$, $\mathrm{z}=0.2 \mathrm{~m}$ ) in a perspective view from the west

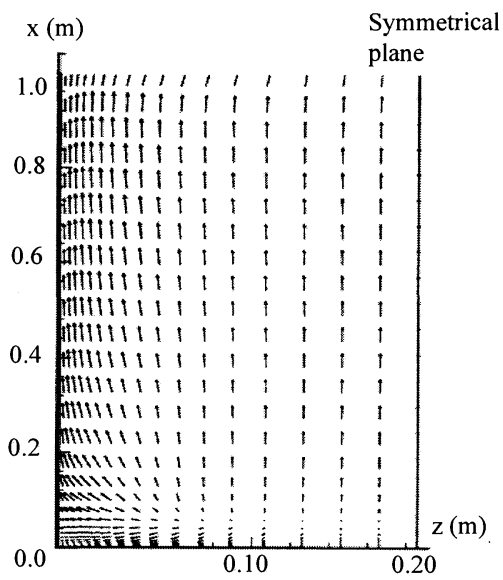

FIGURE 6. Velocity vectors on the plane $(\mathrm{x}, \mathrm{z})$ near the vertical burning wall $(\mathrm{y}=0.01$ $\mathrm{m})$ in a perspective view from the south
FIGURE 5. Temperature contours on the plane $(\mathrm{x}, \mathrm{z})$ near the vertical burning wall $(y=0.01 \mathrm{~m})$ in a perspective view from the south

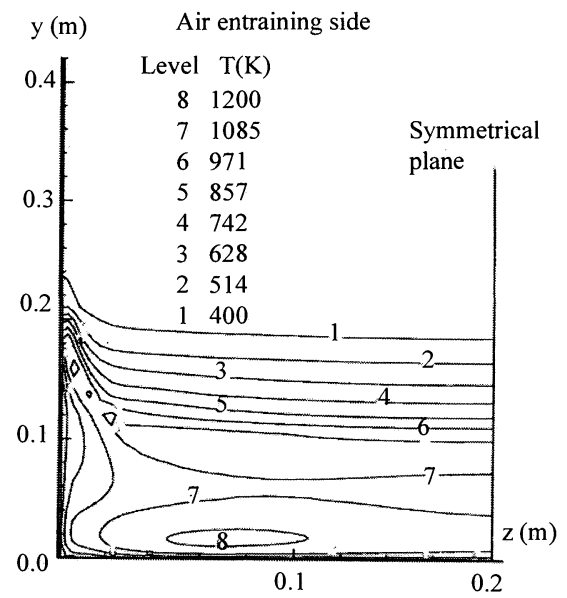

FIGURE 7. Temperature contours on the plane $(y, z)$ just above the rectangular pool fire $(\mathrm{x}=0.015 \mathrm{~m})$ 
the deflection is not generated. However, when drawing near the vertical burning wall $(y<0.2$ $\mathrm{m})$, the temperature of the ambient gas increases progressively with the hottest gas located near the burning wall (north) where the flow is also strongly deflected toward the low corner region.

\section{Capacity and Limitation of the Theoretical Model}

A comparison between the prediction and experiment allows to estimate the capacity and limitation of the theoretical model used here. Even in such a configuration, it is possible to compare prediction and experiment for comparatively few points, and for representative results. Here, only the results on the symmetrical plane $(\mathrm{x}, \mathrm{y}, \mathrm{z}=0.2 \mathrm{~m})$ at various locations $(\mathrm{x}=0.015,0.230,0.470$ and $0.720 \mathrm{~m})$ along the vertical wall in a perspective view from the west (see Fig.1), are compared with the experimental data [8].

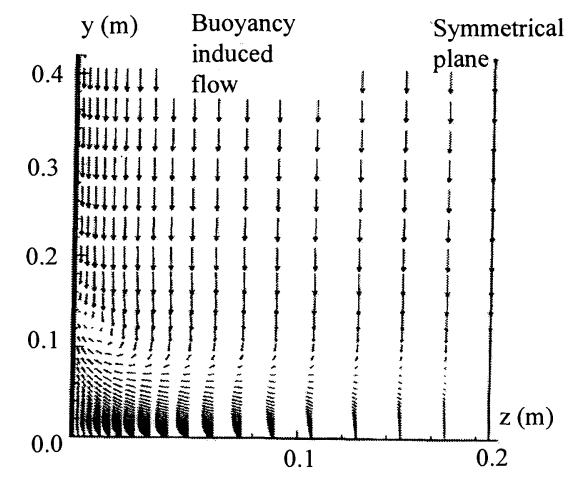

FIGURE 8. Velocity vectors on the plane (y, z) just above the rectangular pool fire $(\mathrm{x}=0.015 \mathrm{~m})$

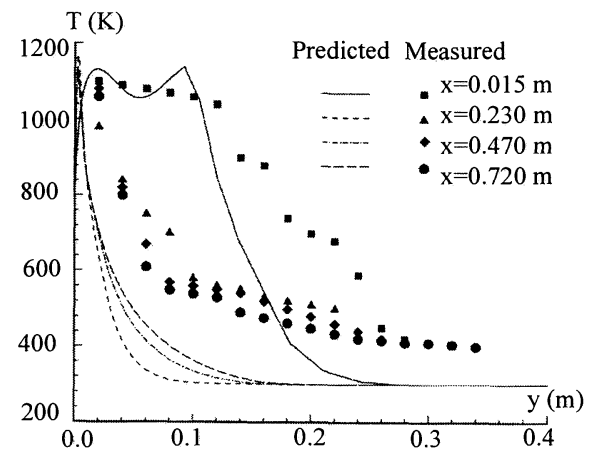

FIGURE 9. Predicted (lines) and experimental (symbols) temperature profiles at various locations along the vertical burning wall

The predicted temperature profiles at various locations along the vertical burning wall are compared with the experimental data in Figure 9. It can be seen that the general shape of the temperature profiles is correct, but that the calculated flame spread is significantly underestimated. The temperature increases with distance to the vertical burning wall from about $800 \mathrm{~K}$ to a maximum of $1100 \mathrm{~K}$, then it roughly decreases beyond the reacting zone, identified by a discontinuity. The peak temperature always occurs nearby the burning wall, and far away from the experimental peak one. Outside of the burning wall region, the predicted temperature is about $20 \%$ smaller than the experimental data. Thus the discrepancies between the measured and calculated temperatures may be due to the use of the flame sheet approximation, which can not be justified near the horizontal burner surface where initial mixture between combustible gases and air is occuring. This effect manifestes itself as a significant reduction of the flame spread at the bottom of the fire, and also all along the vertical burning wall. 
The profiles of the mean axial velocity, $u(\mathrm{~m} / \mathrm{s})$, at various locations along the vertical burning wall are presented in Figure 10. Good agreement between the predictions and experimental data is obtained thanks to the correct description of the dominant buoyancy term in the momentum equation. Since the temperature and velocity fields are strongly coupled for the buoyancy-induced flow, similarly to the temperature profiles, the discrepancy in the velocity profiles is seen between the experiment and prediction. Corresponding to the underprediction of the temperature profiles, the velocity profiles are also always underestimated with the difference equal to $20 \%$. The profiles of the mean transverse velocity, $\mathrm{v}(\mathrm{m} / \mathrm{s})$, are also compared with the experimental data in Figure 11. It should be noted that near the horizontal burner surface at $\mathrm{x}=0.015 \mathrm{~m}$, the entrainment velocity is significantly overestimated. The poor agreement between the measured and predicted values there suggests that entrainment is not well described in this region by the model. The gross overestimation of this transverse velocity seems to be responsible for the larger recirculating zone in comparison with the experimental observation (see Fig. 10 at $\mathrm{x}=0.015 \mathrm{~m}$ ). The reasons for this are as follows.

1. The numerical model : the buoyant instability and the transition to turbulence at the low base can not be taken into account in the simulation.

2. The experiment : the initial momentum above the horizontal combustible surface is very low, and the effects of the buoyant instability on the experimental results increase eventually, identified by a non-realistic cessation of air entrainment in this region (see Fig. 11 at $\mathrm{x}=0.015 \mathrm{~m}$ ).

However, for downstream locations $(x>0.1 \mathrm{~m})$, the transverse velocity profiles are in close agreement with the experimental data, indicating that the entrainment is correctly predicted.

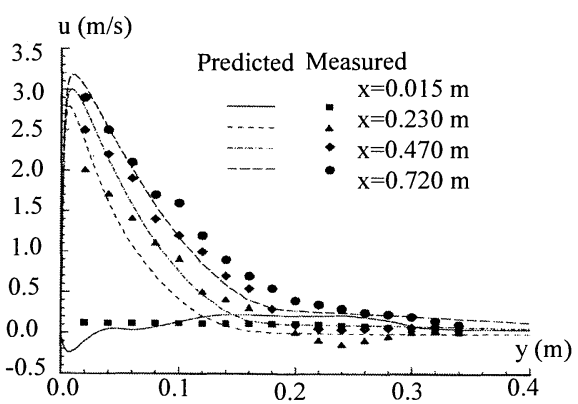

FIGURE 10. Predicted (lines) and experimental (symbols) axial velocity profiles at various locations along the vertical burning wall

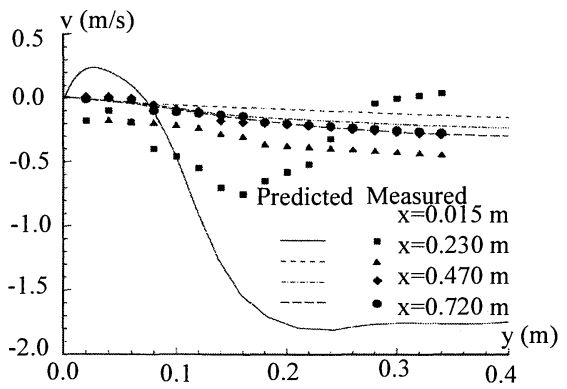

FIGURE 11. Predicted (lines) and experimental (symbols) transverse velocity profiles at various locations along the vertical burning wall

The axial $\left(u^{\prime} u^{\prime}\right)$ and transverse $\left(v^{\prime} v^{\prime}\right)$ velocity fluctuations $\left(\mathrm{m}^{2} / \mathrm{s}^{2}\right)$ are presented in Figures 12 and 13 at various locations along the vertical burning wall. The experimental results show that the axial velocity fluctuation, as shown in Fig.12, near the horizontal burner surface $(x=0.015 \mathrm{~m})$ is relatively weak $\left(0.05 \mathrm{~m}^{2} / \mathrm{s}^{2}\right)$; however, far downstream away from the pool fire $(x>0.1 \mathrm{~m})$, the increase of the velocity fluctuations is almost brutal, up to $0.7 \mathrm{~m}^{2} / \mathrm{s}^{2}$ at $\mathrm{x}=0.23 \mathrm{~m}$, and then remains rather moderate all along the vertical wall. It means that 
development of the turbulence is strongly controlled by the buoyant instability rather than by the mean flow field. On the contrary, the simulation results show that the axial velocity fluctuation ( $u^{\prime} u^{\prime}$ ) is progressively raised up, and the position of its maximum value is shifted away from the vertical burning wall; the higher axial velocity fluctuation is close to the region, where the shear stress is strong due to only the strong velocity and temperature gradients there. It appears, therefore, that in the presence of a strong buoyant instability, conventional buoyancy modifications $[1-3,5-8]$ to the $\mathrm{k}-\varepsilon$ model, derived using the gradient diffusion hypothesis, are incapable of yielding realistic predictions of all components of the
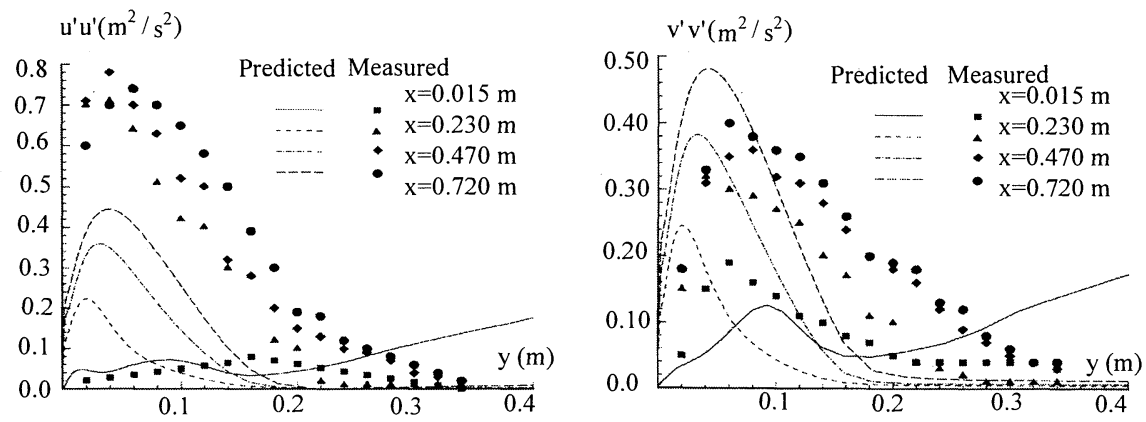

FIGURE 12. Predicted (lines) and experimental (symbols) profiles of the axial velocity fluctuation at various locations along the vertical burning wall

FIGURE 13. Predicted (lines) and experimental (symbols) profiles of the transverse velocity fluctuation at various locations along the vertical burning wall

Reynolds and turbulent scalar flux, all of which are significant in the flow of interest. Comparatively, good agreement (see Fig. 13) between the predictions and experimental data for the transverse velocity fluctuations, $v^{\prime} v^{\prime}$, is obtained. This is due to the fact that effects of the buoyant instability are stronger on the axial velocity than on the transverse one. It is seen that the difference for the transverse velocity fluctuation, v'v', is particularly noticeable at the low part of the vertical wall. Outside of this region, the prediction of the transverse velocity fluctuation, $v^{\prime} v^{\prime}$, seems to be improved, but the higher value of the velocity fluctuation is always located over a narrower region in comparison with the distribution of the experimental data.

\section{CONCLUSION}

The use of the fully elliptic form of three-dimensional model allows to quantify the flame shape and flow field resulting from a vertical burning wall adjacent to a rectangular pool fire that were previously predicted from a single vertical wall or a pool fire but not readily described by a mathematical analysis. What is interesting is that in the presence of a pool fire, the flame thickness at the base of the vertical wall is initially important, and remains practically constant $\left(\mathrm{d}_{\mathrm{f}} \approx 0.07 \mathrm{~m}\right)$ all along the vertical burning wall. As a consequence, buoyancy effects at the base of the vertical burning wall are significant, and the flow is strongly upward accelerated up to $1.73 \mathrm{~m} / \mathrm{s}$ only at a height of $0.1 \mathrm{~m}$. Then, at distant downstream locations, the acceleration of the flow becomes progressive with the velocity 
vectors parallel to the vertical burning wall. Of particular interest is also that a diffusion flame is stabilised thanks to a remarkable recirculating flow over a larger zone just above the horizontal burner surface. However, far downstream away from the pool fire, a rapid broadening of the buoyant gas column appears responsible of the strong narrowing of the buoyantly induced plume.

Further work needs to be performed in order to predict the mechanism generating the buoyant instability and the transition to turbulence. Therefore, caution should be required in applying the common $\mathrm{k}-\varepsilon-\mathrm{g}$ turbulence model based on the gradient diffusion hypothesis to the buoyantly dominated flow. For this, a realistic treatment of the coupling between turbulence and reaction kinetic mechanisms is required. Also some uncertainty exists in the behavior of the flame and of the flow with radiation and soot production. Ongoing work is considering these new physical aspects.

\section{REFERENCES}

1. Kennedy, L. A., "Turbulent Natural Convection Diffusion Flames", $\underline{\text { Heat Transfer and }}$ Turbulent Buoyant Convevtion, II, 683-690, 1977.

2. Kent, J. H. and Bilger, R. W., "The Prediction of Turbulent Diffusion Flame Fields and Nitric Oxide Formation", Sixteenth Symposium on Combustion, The Combustion Institute, Pittsburgh, 1643-1656, 1977.

3. Tamanini, F., "A Numerical Model for the Prediction of Radiation-Controlled Turbulent Wall Fires", Seventeenth Symposium on Combustion, The Combustion Institute, Pittsburgh, 1075-1085, 1979.

4. Delichatsios, M. A., "A Simple Algebraic Model for Turbulent Wall Fires", Rep. RC86BT2, FMRC, 1986.

5. Wang, H. Y., Joulain P. and Most J. M., "Three-Dimensional Modeling and Parametric Study of Turbulent Burning along the Walls of a Vertical Rectangular Channel", Combust. Sci. Tech., 109, 287-307, 1995.

6. Grauford, N. L., Liew, S. K. and Moss, J. B., "Experimental and Numerical Simulation of a Buoyant Fire", Combust. Flame, 61: 63-77, 1985.

7. Adiga, K. C. and Ramaker, D. E., "Modeling Pool-like Gas Flames of Propane", Fire Safety Journal, 14, 241-250, 1989.

8. Annarumma, M. O., "Modélisation Numérique et Validation Expérimentale des Flammes de Diffusion Turbulentes Dominées par les Effets de Gravité", Ph.D. Thesis, 276, Université de Poitiers, France, 1989.

9. Delichatsios, M. A., "Transition from Momentum to Buoyancy-Controlled Turbulent Jet Diffusion Flames and Flame Height Relationships", Combust. Flame, 92: 349-364, 1993.

10. Launder, B. E. and Spalding, D. B., "Mathematical Models of Turbulence", Academic Press, London, 1972.

11. Ince, N. Z. and Launder, B. E., "On the Computation of Buoyancy-Driven Turbulent Flows in Rectangular Enclosures", Int. J. Heat and Fluid Flow, 10: 2, 110-117, 1989.

12. Patankar, S. V., "Numerical Heat Transfer and Fluid Flow", Intertext Books, Ed. McGraw Hill, New York, 1980.

13. Van Doormaal, J. P. and Raithby, G. D., "Enhancements of the SIMPLE Method for Predicting Incompressible Fluid Flows", Numerical Heat Transfer, 147-163, 1984. 